Research paper

\title{
In vitro and ex vivo methods predict the enhanced lung residence time of liposomal ciprofloxacin formulations for nebulisation
}

\author{
Hui Xin Ong a,b, Faiza Benaouda ${ }^{\text {c }}$, Daniela Traini ${ }^{\mathrm{a}, \mathrm{b}}$, David Cipolla ${ }^{\mathrm{d}}$, Igor Gonda ${ }^{\mathrm{d}}$, Mary Bebawy ${ }^{\mathrm{e}}$, \\ Ben Forbes ${ }^{c}$, Paul M. Young ${ }^{\mathrm{a}, \mathrm{b}, *}$ \\ a Respiratory Technology, Woolcock Institute of Medical Research, Glebe, Australia \\ ${ }^{\mathrm{b}}$ Discipline of Pharmacology, Sydney Medical School, University of Sydney, Australia \\ 'Institute of Pharmaceutical Science, King's College London, London, UK \\ ${ }^{\mathrm{d}}$ Aradigm Corporation, Hayward, CA, USA \\ ${ }^{\text {e }}$ School of Pharmacy, Graduate School of Health, University of Technology Sydney, Broadway, Australia
}

\section{A R T I C L E I N F O}

Article history:

Received 29 April 2013

Accepted in revised form 21 June 2013

Available online 10 July 2013

\section{Keywords:}

Isolated perfused lung

Ciprofloxacin

Liposomes

Calu-3

Pulmonary model

Transport

\begin{abstract}
A B S T R A C T
Liposomal ciprofloxacin formulations have been developed with the aim of enhancing lung residence time, thereby reducing the burden of inhaled antimicrobial therapy which requires multiple daily administration due to rapid absorptive clearance of antibiotics from the lungs. However, there is a lack of a predictive methodology available to assess controlled release inhalation delivery systems and their effect on drug disposition. In this study, three ciprofloxacin formulations were evaluated: a liposomal formulation, a solution formulation and a 1:1 combination of the two (mixture formulation). Different methodologies were utilised to study the release profiles of ciprofloxacin from these formulations: (i) membrane diffusion, (ii) air interface Calu-3 cells and (iii) isolated perfused rat lungs. The data from these models were compared to the performance of the formulations in vivo. The solution formulation provided the highest rate of absorptive transport followed by the mixture formulation, with the liposomal formulation providing substantially slower drug release. The rank order of drug release/transport from the different formulations was consistent across the in vitro andex vivo methods, and this was predictive of the profiles in vivo. The use of complimentary in vitro and ex vivo methodologies provided a robust analysis of formulation behaviour, including mechanistic insights, and predicted in vivo pharmacokinetics.
\end{abstract}

(C) 2013 Elsevier B.V. All rights reserved.

\section{Introduction}

Respiratory administration is a well-established approach for targeting drug delivery to treat respiratory diseases and offers many advantages over other routes of administration. The direct application of drugs into the respiratory tract provides rapid onset of action, lower systemic exposure and subsequently reduced side effects [1]. Hence, it is not surprising that inhalation antibiotics have become a standard part of treatment for cystic fibrosis (CF), where recurrent pulmonary infection, especially with Pseudomonas aeruginosa, is the main cause of morbidity and mortality [2,3]. While nebulised antibiotics such as tobramycin (TOBI ${ }^{\circledR}$ ) and aztreonam (Cayston ${ }^{\circledR}$ ) have proven to be an effective therapy against respiratory tract infections in CF patients, these locally acting treatments are rapidly cleared from the lungs. To maintain sufficiently high levels of antibiotics for antimicrobial efficacy, multiple doses

* Corresponding author. Woolcock Institute of Medical Research, 431 Glebe Point Road, Glebe, NSW 2037, Australia. Tel.: +61 29114 0350; fax: +61 291140014.

E-mail address: paul.young@sydney.edu.au (P.M. Young). of high concentration antibiotics with lengthy administration times are required each day.

A sustained release liposomal ciprofloxacin formulation was developed recently with the aim of reducing the burden of therapy by prolonging the residence time of drug in the lungs to reduce the necessity for frequent administration. Additional potential benefits include improved antimicrobial efficacy by maintaining high antibiotic levels throughout the dosing interval, decreasing systemic toxicity and improving local tolerability by avoiding excessive peak concentrations of antibiotics in the airways [4-7]. The liposomal formulation is a liquid nanosuspension of ciprofloxacin formulated with lipids and cholesterol and delivered via nebulisation. Currently, there is no regulatory approved aerosolised form of ciprofloxacin for human use. Chronic use of oral and parenteral ciprofloxacin is limited by its systemic toxicity and by concerns over the emergence of ciprofloxacin resistance in the community. Thus, inhaled liposomal ciprofloxacin is an ideal candidate for chronic prophylactic management of recurrent respiratory infections as it has an established broad-spectrum activity and may be effective in preventing pulmonary exacerbations associated with respiratory tract infections, especially those caused by $P$. aeruginosa. 
Despite the significant interest and research in the field of controlled release products for inhalation, to date, there are no such treatments on the market. The lack of standardised methodology available to predict drug transport from potential controlled release inhalation delivery systems may, in part, have contributed to the absence of such formulations, be it in the form of dry powders, pressurised metered dose inhalers or nebulised formulations $[8,9]$. The disposition of drugs delivered to the lung is a complex phenomenon that depends on the deposition pattern of the drug formulation and the impact of the various mechanisms of distribution and elimination (release, absorption, phagocytosis, metabolism, mucociliary clearance) $[10,11]$. In the past, drug transport from experimental formulations has been investigated using one of a multitude of methods, which are based on different principles and assumptions [12]. This makes comparison between the different controlled release formulations challenging and predictions of behaviour in humans unreliable because none of the in vitro methods mimic fully the disposition mechanisms.

Further, non-primate animals have different anatomies and physiologies of the respiratory tract compared to humans. Disease condition can, of course, add another important dimension that is difficult, if not impossible, to simulate in in vitro, or even in animal, models. Nevertheless, reductionist approaches can provide insights into the mechanisms of release and absorption that may be particularly useful in early product development and mitigate certain risks prior to investigations in humans. Drug release from pulmonary dispersed systems, such as liposomes, is often evaluated using simplified models [13-15]. A typical example is the membrane diffusion method in which the carrier-dispersed phase is suspended in a small volume of continuous phase, separated from a large sink phase by a dialysis membrane that is permeable to the drug. This apparatus is clearly not consistent anatomically with the in vivo physiology encountered by inhaled dosage forms. Normal airways are lined with approximately $\sim 1 \mu \mathrm{L}$ per $\mathrm{cm}$ of liquid, consisting predominantly of water, endogenous phospholipids and mucus [16]. In addition, the normal mucosal airway $\mathrm{pH}$ is slightly acidic ( $\sim \mathrm{pH} 6.6$ ) compared to the blood which has a pH of 7.4 [17].

The characteristics of drug release from dispersed systems are strongly influenced by the biological environment whereby interactions of particles with biological components, such as phospholipids and protein, could significantly alter the release rate of the drug. Therefore, in vitro models of the airway epithelium are being utilised as representative models of the lungs for studying the impact of formulation on respiratory release profiles [18-20]. In such studies, air interfaced airway epithelial cells, such as Calu-3 cells $[18,21]$, are typically used to model the respiratory mucosal surface, and a device for delivering a pharmaceutically-relevant aerosol, such as the twin stage impinger, is used to administer the formulation. However, cell culture systems do not possess the intact structure, the full absorptive pathway or the full spectrum of influences on drug disposition that are present in the human lungs. To study the kinetics of drug transport in the intact target organ, an isolated perfused lung (IPL) model can be used [21]. The IPL is an ex vivo model which possesses the structural integrity and functionality of the lung tissue and, in comparison with in vivo methods, allows the investigation of drug absorption from the lungs without the confounding contributions of oral systemic absorption, distribution and elimination. Consequently, it allows the researcher to elucidate the release kinetics of encapsulated drug and subsequent absorption resulting from the interaction of particles with the pulmonary environment [22].

The aim of the current study was to investigate the impact of a liposomal ciprofloxacin controlled release formulation on drug transport in the lungs using complimentary in vitro techniques and an ex vivo IPL model. The results were analysed with reference to in vivo studies performed in mice using the same formulation
[23]. This provides a rare illustration of the combined ability of the non-clinical model systems to predict and explain the absorption profiles of a sustained release formulation from the lungs. The kinetic profiles of the liposomal ciprofloxacin formulation are compared to ciprofloxacin solution formulation across a series of in vitro, ex vivo and in vivo methodologies, and the correlation and advantages/disadvantages of each are appraised.

\section{Materials and methods}

\subsection{Materials}

Calu-3 cells were purchased from the American Type Cell Culture Collection (ATCC, Rockville, US). Nonessential amino acids solution, trypan blue solution $(0.4 \% \mathrm{w} / \mathrm{v})$ and CelLytic ${ }^{\mathrm{TM}} \mathrm{M}$ Cell Lysis, protease inhibitor cocktail, were purchased from SigmaAldrich (Sydney, Australia). Other cell culture reagents including trypsin-EDTA solution (2.5 g/L trypsin, $0.5 \mathrm{~g} / \mathrm{L}$ EDTA), Dulbecco's Modified Eagle's Medium (DMEM, without phenol red and L-glutamine, including sodium bicarbonate and $15 \mathrm{mM}$ HEPES), Phosphate buffered saline (PBS), L-glutamine solution (200 mM), foetal bovine serum (FBS) and Hank's balanced salt solution (HBSS) were obtained from Invitrogen (Sydney, Australia). Transwell cell culture inserts $\left(0.33 \mathrm{~cm}^{2}\right.$ polyester, $0.4 \mu \mathrm{m}$ pore size) were purchased from Corning Costar (Lowell, MA, USA), and all other sterile culture plastic wares were from Sarstedt (Adelaide, Australia). Salts including $\mathrm{NaCl}, \mathrm{KCl}, \mathrm{CaCl}_{2}, \mathrm{MgSO}_{4}, \mathrm{NaHCO}_{3}, \mathrm{KH}_{2} \mathrm{PO}_{4}$, D-glucose, 4-(2-hydroxyethyl)piperazine-1-ethanesulfonic acid (HEPES) and heparin were purchased from Sigma-Aldrich (Poole, UK). All solvents used were of analytical grade and were supplied by Biolab (Victoria, Australia).

\subsection{Preparation of liposomal and solution ciprofloxacin formulations}

The preparation of the ciprofloxacin formulations have been reported previously [20]. Briefly, liposomal ciprofloxacin, $50 \mathrm{mg} / \mathrm{mL}$, is an aqueous liposome formulation comprised of lipids and cholesterol. It was prepared by an active loading method through a transmembrane $\mathrm{pH}$ gradient, generated in response to an ammonium sulphate gradient $[23,24]$. Free ciprofloxacin, $20 \mathrm{mg} / \mathrm{mL}$, is an acetate-buffered aqueous formulation at $\mathrm{pH}$ 3.3. To produce the mixture formulation, equal concentrations of the liposomal and solution formulations were added together immediately prior to the experiments to produce a concentration of 50:50 (\% w/w).

\subsection{Ciprofloxacin analysis}

The HPLC system used to measure ciprofloxacin concentrations in the isolated perfused lung samples consisted of a SpectraSYSTEM UV1000 UV-vis detector (Thermoseparation Products, Salford, UK), JASCO PU-980 HPLC pump (Jasco, Tokyo, Japan), SpectraSYSTEM AS3000 Autosampler (Thermoseparation Products, Salford, UK) and an Optimal ODS-H column ( $3 \mu \mathrm{m}, 150 \times 4.6 \mathrm{~mm}$, Capital HPLC Limited, Broxburn, West Lothian, Scotland). The mobile phase was a mixture of methanol and $0.1 \mathrm{M}$ sodium dihydrogen phosphate, at a 30:70 (\% v/v) ratio, with $\mathrm{pH}$ adjusted to 3.35 with phosphoric acid. The flow rate was set to $0.8 \mathrm{~mL} / \mathrm{min}$, and $50 \mu \mathrm{L}$ of each sample was injected into the column. The UV detector was set to $275 \mathrm{~nm}$. Linearity of ciprofloxacin was obtained between 0.1 and $50 \mu \mathrm{g} / \mathrm{mL}\left(R^{2}=0.99\right)$, with a retention time of $14.6 \mathrm{~min}$.

Quantitation of ciprofloxacin for samples from all other experiments was performed using the HPLC method as previously described $[18,20]$. To determine the total ciprofloxacin concentration, the liposomal and mixture formulation samples required 
pretreatment prior to HPLC analysis, to rupture the liposomes and separate the drug from the lipids. This was accomplished by dilution with methanol to a concentration of $80: 20(\% \mathrm{v} / \mathrm{v})$ methanol:water. The samples were then centrifuged at $10,000 \mathrm{~g}$ for 5 min to allow sedimentation of the lipids. The supernatant was collected and further diluted $10 \times$ with deionised water, prior to quantification by high-performance liquid chromatography (HPLC).

\subsection{Dialysis membrane diffusion}

Dialysis membranes (MW cut-off 6000-8000, Cellu.Sep, Texas, USA) were soaked in deionised water for $20 \mathrm{~min}$ prior to use to ensure thorough wetting of the membrane. The pore size of the dialysis bag was chosen to allow free diffusion of ciprofloxacin into the sink phase and to prevent the exit of the liposomal nanoparticles. This study was carried out in phosphate buffered saline (PBS) at $\mathrm{pH} 7.4$ over the same 240 min period of time used for the invitro cell model studies. The liposomal and mixture formulations $(1 \mathrm{~mL})$ were enclosed in the dialysis bag, which was immersed in $200 \mathrm{~mL}$ of PBS solution to ensure sink conditions. The solutions were maintained at $37^{\circ} \mathrm{C}$ in a water bath under constant agitation using a magnetic stirrer (300 rpm). Samples of $600 \mu \mathrm{L}$ were withdrawn at pre-determined time points $(5,10,15,30,120,150$, 180 and $240 \mathrm{~min}$ ) from the incubation medium and were replaced with equal volumes of fresh phosphate buffer solutions (PBS). Control experiments were also performed to determine the release behaviour of the drug from the solution formulation using the same procedure. All experiments were performed in triplicate.

\subsection{Absorptive profiling in vitro}

Calu- 3 cells were grown at the air interface, as described previously $[20,25]$. Experiments were performed between day 11 and 14, from passages 35-42. Deposition of aerosolised formulations onto the Calu-3 cells was performed using a modified glass twin stage impinger apparatus (TSI; British Pharmacopoeia Apparatus A, Copley Scientific, UK) that accommodates a transwell insert located at the end of the jet in the second lower chamber (absence of solution). The set-up of the apparatus is described in detail elsewhere $[18,26]$. The PARI LC Sprint ${ }^{\circledR}$ nebuliser, powered by the PARI TurboBoy ${ }^{\circledR} S$ compressor, was fitted to the entrance of the TSI using a custom-made mouthpiece adapter, and the experimental method followed that of Ong et al. [20]. Briefly, $2 \mathrm{~mL}$ of the mixture formulation $(2.5 \mathrm{mg} / \mathrm{mL})$ was nebulised for $5 \mathrm{~s}$ at a flow rate of $15 \mathrm{~L} / \mathrm{min}$ to allow $<20 \mu \mathrm{g}$ of ciprofloxacin to be deposited onto the Calu-3 cells during the formulation.

Immediately after deposition, the Transwell insert was removed, the outer surface was wiped dry to remove any external adhering droplets, and the insert was placed into a well of a 24well plate containing $600 \mu \mathrm{L}$ of pre-warmed HBSS (receiver fluid). Samples were transferred to a new well containing fresh HBSS at pre-determined time points (up to $4 \mathrm{~h}$ ) to maintain sink conditions. At the final time point, cells were gently washed with HBSS to collect the remaining drug on the cell surface and subsequently harvested and lysed on ice in CellLytic ${ }^{\mathrm{TM}} \mathrm{M}$ Cell Lysis reagent with $1 \%(\mathrm{v} / \mathrm{v})$ protease inhibitor cocktail, according to manufacturer's recommendation to quantify intracellular drug. Lysates were centrifuged at $10,000 \mathrm{~g}$ for $10 \mathrm{~min}$ at $4{ }^{\circ} \mathrm{C}$. The supernatant was then aspirated and diluted to an appropriate volume. Samples were analysed by HPLC. The sum of the drug that was transported across the epithelial cells and the drug recovered from the surface and 'inside' the cells after the $4 \mathrm{~h}$ period provided an estimate of the quantity of ciprofloxacin deposited on the cells.

Assessment of the Calu-3 monolayer's integrity was performed using transepithelial electrical resistance (TEER) measurements, as previously described [18,27], using an epithelial voltohmmeter (EVOM, World Precision Instruments, USA). TEER measurements were performed on untreated-control cells and after the deposition experiments were complete.

\subsection{Isolated perfused lung}

Lungs were obtained from male Wistar rats weighing 220$300 \mathrm{~g}$ (Harlan UK Ltd., UK). Rats were anesthetised by intraperitoneal injection of pentobarbitone $(1.2 \mathrm{~mL} / \mathrm{kg}$ of a $200 \mathrm{mg} / \mathrm{mL}$ solution). The lungs were excised and perfused through the pulmonary artery on the basis of a method previously described [28]. Briefly, after the tracheal intubation, the lung was inflated with $1.5 \mathrm{~mL}$ air. The abdomen was opened and heparin (500 international units [IU], Leo Ltd., UK) injected into the vena cava. The animals were then exsanguinated by withdrawal of blood from the inferior vena cava, and the thorax was opened. A cannula was inserted into the pulmonary artery and held in place by tightening a ligature previously placed around the pulmonary artery and aorta. The lower parts of the ventricles were cut to allow free efflux of the perfusate. Perfusion, in a single pass mode, was commenced with modified Krebs buffer (composition mmol/L: $\mathrm{NaCl}$ $118 \mathrm{mM}, \mathrm{KCl} 4.7 \mathrm{mM}, \mathrm{CaCl}_{2} 2.5 \mathrm{mM}, \mathrm{MgSO}_{4} 1.2 \mathrm{mM}, \mathrm{NaHCO}_{3}$ $24.9 \mathrm{mM}, \mathrm{KH}_{2} \mathrm{PO}_{4} 1.2 \mathrm{mM}$, HEPES $10 \mathrm{mM}$, D-glucose $11 \mathrm{mM}$ ). The lungs, heart and trachea were then removed and suspended over a beaker at room temperature during the drug absorption study. Krebs buffer was held in a heated $\left(37^{\circ} \mathrm{C}\right)$ reservoir and saturated with $95 \%$ oxygen: $5 \%$ carbon dioxide mixture and the perfusion flow rate were maintained at ca. $8 \mathrm{~mL} / \mathrm{min}$ using a peristaltic pump (Masterflex, UK). The effluent perfusate was collected, and $5 \mathrm{~mL}$ aliquots were removed at different time points.

\subsection{Absorptive profiling ex vivo}

After isolation, the lung preparation was allowed to stabilise for 2-3 min before delivery of the formulations. The formulations were diluted with PBS solution to a ciprofloxacin concentration of $5 \mathrm{mg} / \mathrm{mL}$. A volume of $100 \mu \mathrm{L}$ of each diluted formulation was instilled into the lungs (equivalent to $500 \mu \mathrm{g}$ of ciprofloxacin) through the tracheal cannula using a $10 \mathrm{~mL}$ syringe and chased by approximately $2 \mathrm{~mL}$ of air to distribute the solution and re-inflate the lungs. Air volume in the lungs was kept constant; perfusion flow was stable throughout the experiment and was not influenced by drug administration. This method of administration provides precise control of the dose, minimising drug loss in the instrumentation and bypassing nasal and oropharyngeal deposition. Perfusion buffer that dripped from the left atrium was sampled at predetermined time points $(1,2,3,4,5,6,7,8,9,10,12,15,17,20$ and $25 \mathrm{~min}$ ). The tissue viability was verified by visual inspection and for signs of oedema and by determination of the wet to dry weight ratios after incubation of the lung at $80^{\circ} \mathrm{C}$ for $24 \mathrm{~h}$.

Samples collected were treated by diluting with equal volumes of methanol. Subsequently, the samples were vortexed and centrifuged at $13,000 \mathrm{rpm}$ for $20 \mathrm{~min}$. The supernatant was collected for quantification of ciprofloxacin by HPLC analysis. For the liposomal and mixture formulations, $2 \mathrm{~mL}$ of samples was left to dry overnight. The samples were then rinsed with $0.4 \mathrm{~mL}$ for the liposomal formulation or $1 \mathrm{~mL}$ for the mixture formulation, using 50:50 (\% v/ v) methanol:water. This was performed to increase the drug concentration in the samples for quantification.

\subsection{Data analysis}

The drug mass transport (\% absorbed) from the airways to the perfusate during the $30 \mathrm{~min}$ IPL experiments was calculated as the mass fraction of the given dose recovered from the perfusate. 
Similar calculations were performed with the transport experiments on the air interface Calu- 3 cell model, whereby the drug mass transport from the Calu- 3 was calculated as the cumulative mass fraction of the total drug recovered throughout the $4 \mathrm{~h}$ experiment as previously described [18]. For the dialysis membrane diffusion experiment, drug concentrations were measured and expressed in terms of per cent total recovery. All data were subsequently plotted as mean cumulative percentages ( \pm standard deviation) of drug release/transport versus time.

The ciprofloxacin kinetics from the different methodologies were fitted to mathematical models that describe drug dissolution and diffusion functions, specifically Zero order, First order, Higuchi and Hixon-Crowell. The linearised forms of these functions were evaluated using $R^{2}$ regression analysis. Of course, it must be appreciated that a good numerical fit is not necessarily a confirmation of a specific physical mechanism and that the goodness of fit depends on the extent of release observed [29].

\section{Results}

\subsection{Dialysis membrane diffusion}

The release profile of each formulation across a dialysis membrane was studied in PBS at $\mathrm{pH} 7.4$ and $37^{\circ} \mathrm{C}$. Over the $4 \mathrm{~h}$ experiment, the solution formulation demonstrated rapid ciprofloxacin diffusion from the dialysis bag with $>96 \%$ of the drug released (Fig. 1), suggesting no retardation of drug diffusion across the dialysis membrane. The release profiles from the liposomal and mixture formulations suggested sustained release of ciprofloxacin. The release over the $4 \mathrm{~h}$ period was approximately $8 \%$ and $33 \%$ of ciprofloxacin from the liposomal and mixture formulations, respectively.

\subsection{Absorptive profiling in vitro}

The total amount of ciprofloxacin deposited onto the Calu-3 cells from the three formulations was equivalent (11.14 \pm $3.47 \mu \mathrm{g}, p>0.05$ ). However, each formulation displayed different kinetic profiles (Fig. 2). The liposomal ciprofloxacin formulation presented a sustained release profile of antibiotic, with only $1.5 \%$ of the drug being transported across the epithelia over a $4 \mathrm{~h}$ period. In comparison, the solution formulation delivered a more rapid transport rate of ciprofloxacin with $>33 \%$ of the drug being absorbed across the cells. The mixture formulation showed an intermediate transport rate, with approximately $7 \%$ of the drug being transported across the Calu- 3 .

The deposition of the nebulised formulations on the epithelial cells using the modified TSI did not produce a detrimental effect

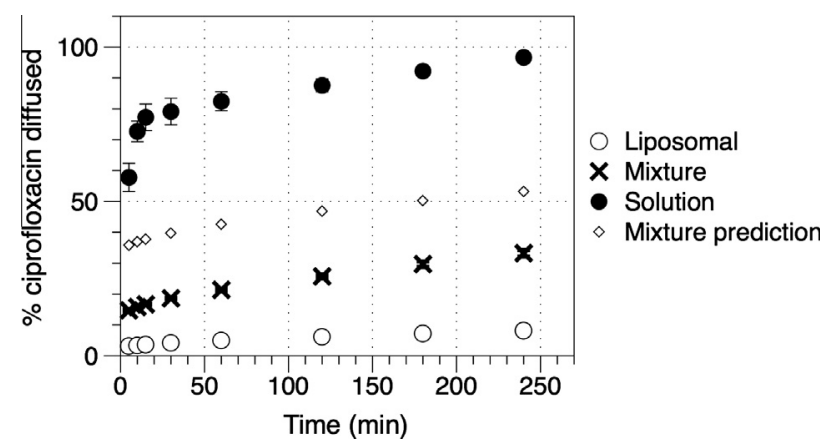

Fig. 1. In vitro drug release profiles of ciprofloxacin across a dialysis membrane from the ciprofloxacin solution ( $\mathrm{SD}<5 \%$ ), mixture formulation $(\mathrm{SD}<1.1 \%)$ and liposomal ciprofloxacin $(\mathrm{SD}<1.5 \%)(n=3$, mean \pm SD).

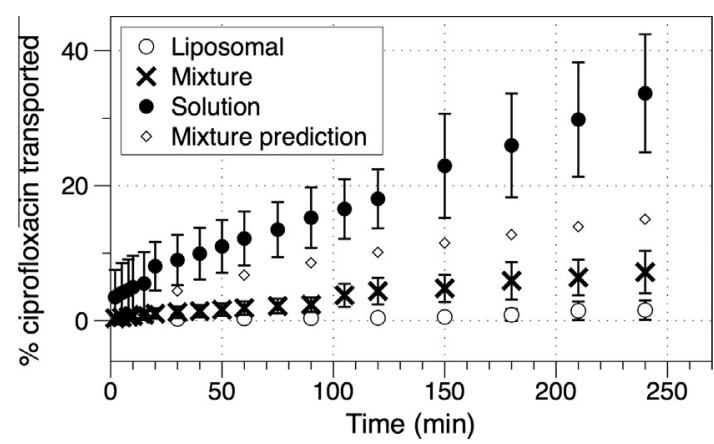

Fig. 2. Cumulative absorptive ciprofloxacin transport after deposition of aerosolised solution, mixture and liposomal formulations to the surface of air interface cultured Calu-3 epithelial cell layers $(n \geq 3$, mean \pm SD).

on the integrity of the cells under the conditions and timescale studied, as measured by transepithelial electrical resistance which remained unchanged from the control at $548 \pm 3 \Omega \mathrm{cm}^{2}(n=3$, $p>0.05$ ).

\subsection{Isolated perfused lung model}

Discrete absorptive profiles of drug into the perfusate were measured from each of the ciprofloxacin formulations (Fig. 3). Over $25 \mathrm{~min}, 82 \pm 5.5 \%$ of the delivered dose of the free ciprofloxacin formulation was transported into the perfusate. In comparison, the liposomal formulation resulted in a slower drug transport, with the cumulative amount of ciprofloxacin transported being $12.3 \pm 1.8 \%$. Administration of the mixture formulation resulted in $33.1 \pm 4.3 \%$ of the ciprofloxacin being transported into the perfusate in $25 \mathrm{~min}$, presumably reflecting principally the rapid absorptive clearance of the free ciprofloxacin component. No lung oedema was present during any of the experiments; determined visually or by weight.

\subsection{Release kinetics of ciprofloxacin from nebuliser formulations}

Distinct kinetic profiles from the liposomal and solution formulations were obtained across all methodologies. The kinetics of the solution formulation were dependent on concentration (i.e. the rate of transport/diffusion decreased as time increased). In comparison, the liposomal formulation demonstrated an approximately linear profile with time. The profiles obtained for the mixture formulation varied according to the methodologies, suggesting that a method-dependent impact on the observed transport rates. Conventional, mathematical models of drug

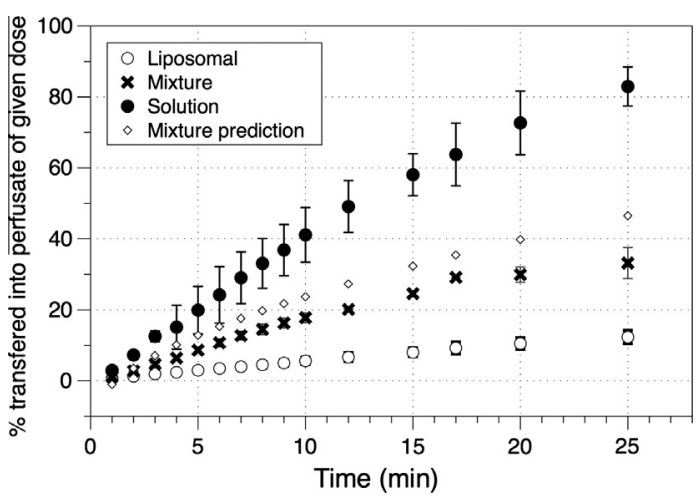

Fig. 3. Cumulative percentage of ciprofloxacin transferred to the perfusate vs. time in the isolated perfused rat lung model following intratracheal administration of $100 \mu \mathrm{L}$ of ciprofloxacin $5 \mathrm{mg} / \mathrm{mL}$ formulations $(n=4$, mean \pm SD). 
Table 1

The absorptive half-life of ciprofloxacin delivered in the liposomal and solution formulations, estimated using the best-fit mathematical model.

\begin{tabular}{|c|c|c|c|c|c|}
\hline & \multirow[t]{2}{*}{ Best-fit model } & \multicolumn{4}{|l|}{ Half-life, $t_{1 / 2}(\min )$} \\
\hline & & Calu-3 & Dialysis bag & Isolated perfused lung (IPL) & In vivo mice lung ${ }^{a}$ \\
\hline Free ciprofloxacin (FCI) & Higuchi & $723 \pm 319\left(R^{2}=0.964\right)$ & $39 \pm 28\left(R^{2}=0.84\right)$ & $12.7 \pm 2.1\left(R^{2}=0.990\right)$ & $7.8^{\mathrm{b}}$ \\
\hline Liposomal ciprofloxacin (CFI) & Zero order & $16031 \pm 11,549\left(R^{2}=0.872\right)$ & $2255 \pm 422\left(R^{2}=0.982\right)$ & $99.2 \pm 15.3\left(R^{2}=0.996\right)$ & $697\left(R^{2}=0.982\right)$ \\
\hline
\end{tabular}

a From Ref. Yim et al. [23].

b Estimated from the first two points of the free ciprofloxacin data as $>95 \%$ of the drug has been transported across the lungs within the first 15 min (Fig. 4 ).

dissolution/release and diffusion were applied to all data. The bestfit mathematical function was selected based on the correlation coefficient criterion, $R^{2}$, and the half-life of the drug for the different formulations was estimated (Table 1). We would like to note, however, that a good fit of data to a particular model is not necessarily a confirmation that the physical models underlying the mathematical equation represent the mechanism of release for a particular formulation, especially if the detailed complete release profile is unavailable [29].

Regression analysis indicated that the data obtained for the solution formulation best fits the Higuchi diffusion model: $r \%=k_{\mathrm{H}-}$ $t^{1 / 2}$ where $r \%$ is the percentage of drug released at time $t$ and $k_{\mathrm{H}}$ is the Higuchi rate constant. In comparison, data from the liposomal formulation best fit the zero-order kinetic equation: $r \%=k t$. For the mixture formulation, no single mathematical function was able to describe the profiles across all the methodologies used, and it was excluded from the half-life calculations. The half-lives calculated generally showed that the Calu-3 epithelial cell model measured the longest half-life, followed by dialysis membrane diffusion, isolated perfused lung and finally in vivo mice lung. An exception to this order is the relative rates of transport of ciprofloxacin from the liposomal formulation in the IPL and in vivo mice lung.

\section{Discussion}

The transport profiles of ciprofloxacin from the different ciprofloxacin formulations were assessed using three different methodologies and were compared to in vivo pharmacokinetic data reported previously [23]. Qualitatively, the kinetic profiles of the formulations with the different methodologies were similar, and this was also true for the relative rank of the rates, with the free ciprofloxacin formulation (FCI) having the highest rate of transport, followed by the mixture formulation and finally the liposomal ciprofloxacin formulation (CFI) across all methodologies. Unfortunately, while this rank order remained consistent across all methodologies, variations in the rates and extent of transport/diffusion were observed between them.

Ciprofloxacin was trapped in liposomes by encapsulation of the drug in an ionised state [24]. The slow transport rates of ciprofloxacin after administration to the in vitro models were consistent with retention of the liposomes in the donor compartment and the slow release of ciprofloxacin as only uncharged molecules migrated across the liposomal membrane bilayer. All methods used could be approximated by a zero-order rate kinetic of the liposomal sustained release formulation in contrast to the solution formulation, which was consistent with Higuchi kinetics (which itself is a good approximation to the planar diffusion equation solution at short times [29,30]). The Higuchi model is indicative of a diffusion-controlled transport process, i.e., fully-dissolved ciprofloxacin diffusing rapidly across the dialysis membrane, or in case of Calu-3 and IPL models spreading laterally over the mucosal surface then diffusing across the lung epithelium [20]. Hence, as concentration in the donor compartment reduces (i.e. in the epithelial lining fluid or inside of the dialysis bag), a reduction in the rate of diffusion across the membrane or epithelium occurs. In comparison, the slow rate of transport/diffusion of ciprofloxacin in the liposomal formulation indicates that a constant rate of release of drug from the liposomes is likely the limiting step (i.e. slower than the rate of diffusion through the membranes), resulting in an apparent zero-order process.

The rationale behind the mixture formulation approach was to rapidly expose the lung to therapeutic levels of antibiotic, via the free component, while prolonging residence time through the liposomal component. This approach of combining the immediately available free ciprofloxacin and the slow release liposomal ciprofloxacin could be tailored to optimise therapeutic efficacy, reduce frequency of administration and reduce the potential for formation of resistant colonies. Interestingly, the rate and extent of ciprofloxacin transported across the Calu- 3 cell line and dialysis membrane was not the predicted mean of the profiles for the individual formulations, and none of the four mathematical models could be employed universally to describe the kinetic profile. This may be attributed to the dual release components of the formulation, where the initial phase is a rapid release from the free component, followed by the contribution of the sustained release of ciprofloxacin from liposomes.

If the mixture formulation followed a dual release profile, a combination formula $C_{t}=50 \%(k t)+50 \%\left(k_{\mathrm{H}} t^{1 / 2}\right)$ based on the bestfit models for the free and liposomal ciprofloxacin formulations would predict its release profile. The observed values fell below the predicted values in all test methods, but to different extents. When the experimental data were compared to predicted values using normalised root mean square deviation (NRMSD), the observed values in the IPL model were closest to the predicted values (NRMSD $=0.20$ ), followed by the Calu- 3 cell model, the dialysis technique with NRMSD of 0.92 and 1.13 , respectively. This suggests that the in vitro models exhibit an artefact not seen in the ex vivo model and in vivo studies, i.e., that the presence of liposomes is affecting both the overall transport extent and the rate in the in vitro models either by interaction of liposomes with the dialysis membrane/epithelium, with the free drug or both. Furthermore, it should be pointed out that while liposomal formulation shows no drug release on storage [31], when administered to animals or humans, it leads to an apparent elimination half-life of 10$12 \mathrm{~h}[4,31]$. The differences observed in the models may possibly be dependent on the proximity between the liposomes and the free drug and the duration of the experiments. The ex vivo model has a larger surface area for drug absorption compared to the in vitro models where the free drug will be at a higher concentration and in closer proximity to the liposomes. Additionally, the longer experimental duration of the in vitro models could have provided additional time for any interactions to occur.

Many studies [32-35] that attempted to establish a correlation between a cell culture system with either an IPL model or in vivo animal studies have used the apparent permeability of the drugs (estimated from flux data), together with the apparent first-order absorption rate constant to establish a relationship. However, in this study, the use of the TSI to deposit aerosol droplets onto the Calu-3 cells instead of the conventional direct application of drug 
solution onto the cell surface has made it difficult to estimate the initial concentration of drug deposited, which is required for the estimation of apparent permeability. This is due to the lack of information regarding the exact volume of surface liquid produced by the air interface Calu-3 cells and the volume of aerosol droplets deposited on the cells. Hence, half-lives for the transport of the formulations based on best-fit kinetic model were used to allow direct comparison of the different formulations and methodologies.

Liposomal and solution formulations displayed a decreasing absorptive half-life rank order where Calu-3 epithelial model > dialysis method > IPL model $>$ in vivo mice lung (with the exception for the liposomal formulation murine lung half-life being greater than that of the IPL model) (Table 1). The absorptive halflife of the liposomal formulation in the IPL study was comparable with the in vivo study in mice, which could be attributed to the preservation of the organ's architecture and full range of cell types that are present in vivo. This includes the presence of transporter proteins such as P-glycoprotein, multidrug resistance associated protein and solute carrier transporters in the lung epithelia cells that may be absent in cell culture models. These transporters may have an impact on the absorption of ciprofloxacin, as transport of this drug has been shown to be mediated by some of these transporters [36-38]. However, limitation to the IPL technique is that it is labour-intensive, requires adept technical expertise for the surgery and perfusion and the limited viability of the lung ex vivo.

Particularly, when assessing controlled release formulations, the information obtained from a partial release profile experimental period may be misleading. Controlled release formulations often exhibit two distinct phases: the initial rapid release of drug due to a burst effect followed by a slower drug release corresponding to the sustained drug release phase. Hence, the initial burst effect may explain the lower absorptive half-life estimated for the liposomal formulation in the IPL method compared to the in vivo study, which was carried out over $24 \mathrm{~h}$. This burst effect was observed in the in vivo study (Fig. 4) whereby estimation of the half-life from the initial two time points showed comparable half-life (129 min) to the IPL study. Although the IPL model may not be suitable for the study of controlled release formulations and its kinetic properties, nevertheless, it provided a good approximation of the absorption half-life for conventional formulations, as seen with the solution formulation.

The kinetic profiles of the different ciprofloxacin formulations observed in the Calu- 3 model were in qualitative agreement with the other methodologies. However, the absorptive half-lives for both formulations calculated in the Calu-3 model were approximately an order of magnitude higher compared to that measured in vivo. The deposition of the ciprofloxacin formulation on a relatively small surface area of the Calu- 3 cell model could contribute to the slower transport in comparison with deposition on the larger surface area available in the lungs. Another contributing factor could be the lower permeability of ciprofloxacin in the Calu- 3 cell model compared to native epithelium. The barrier provided by Calu-3 cells is greater than that of excised human tissues, rat lungs and rabbit airway epithelium [32,39]. Calu-3 cell-derived data have indicated that $2 \%$ of insulin would be systemically absorbed which was lower in comparison with the $5-10 \%$ of insulin absorbed with $\sim 6 \%$ bioavailability reported in human and rat, respectively $[40,41]$. It should be noted that bioavailability reflects all the disposition mechanisms (release, absorption, distribution, metabolism, phagocytosis, mucociliary clearance) that affect encapsulated and unencapsulated drug $[10,42]$ and is not equivalent to the per cent absorbed.

However, the simplicity, robustness and experimental control available with the air interface Calu- 3 model provide a good platform for assessing the initial kinetic profile of controlled release

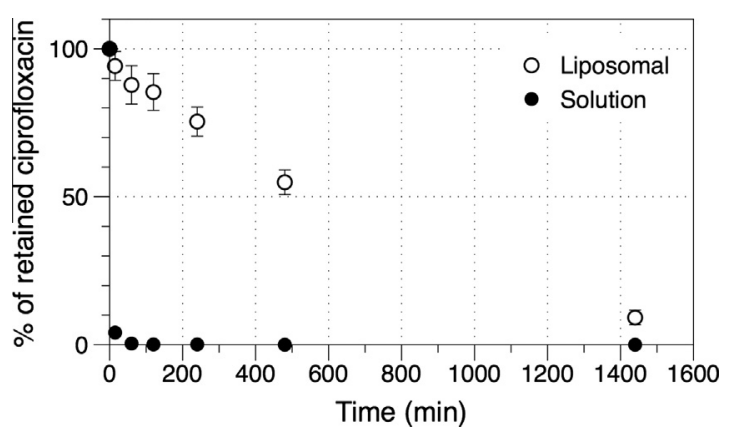

Fig. 4. Retention of ${ }^{14} \mathrm{C}$-ciprofloxacin in the lungs of Balb/C mice post-intranasal instillation of $50 \mu \mathrm{L}$ of ciprofloxacin $1 \mathrm{mg} / \mathrm{mL}$. The lungs of the mice were harvested following sacrifice and processed for liquid scintillation counting to quantify the amount of ciprofloxacin remaining in the lungs. Reproduced with permission from Respiratory Drug Delivery [23].

formulations (e.g. rank-order correlations). And may be better than "tissue" models for identifying individual processes that involve complex and dynamic relationships across different cell types [22].

\section{Conclusions}

Liposomal formulation of ciprofloxacin has the potential to provide a promising new treatment for pulmonary infections. The release of drug from the liposomal formulation was compared to release from a solution formulation in a series of biopharmaceutical models for inhaled drug delivery. The same rank order and a consistent mechanism of drug release/transport (concentrationindependent for the liposomal formulation, diffusion controlled for the solution formulation) were discerned for the formulations using the in vitro andex vivo methods, and this was predictive of the profiles in vivo. The use of complimentary in vitro methodologies provided a robust analysis of formulation behaviour and predicted the in vivo pharmacokinetics. The liposomal formulation provided zero-order release kinetics that prolonged antibiotic residence time in the lungs.

\section{Acknowledgement}

The research leading to these results has received funding from the European Community's Seventh Framework Programme (FP7/ 2007-2013) under grant agreement no. 233533.

\section{References}

[1] D.E. Geller, Aerosol antibiotics in cystic fibrosis, Respir. Care 54 (2009) 658670.

[2] T.S. Murray, M. Egan, B.I. Kazmierczak, Pseudomonas aeruginosa chronic colonization in cystic fibrosis patients, Curr. Opin. Pediatr. 19 (2007) 83.

[3] D. Wood, A. Smyth, Antibiotic strategies for eradicating Pseudomonas aeruginosa in people with cystic fibrosis, Review (2009).

[4] P. Bruinenberg, J.D. Blanchard, D.C. Cipolla, F. Dayton, S. Mudumba, I. Gonda, Inhaled liposomal ciprofloxain: once a day management of respiratory infections, in: R.N. Dalby, P.R. Byron, J. Peart, J.D. Suman, S.J. Farr (Eds.), Respiratory Drug Delivery, Davis Healthcare International Publishing, River Grove, Orlando, Florida, 2010.

[5] J. Gubernator, Z. Drulis-Kawa, A. Dorotkiewicz-Jach, W. Doroszkiewicz, A. Kozubek, In vitro antimicrobial activity of liposomes containing ciprofloxacin, meropenem and gentamicin against gram-negative clinical bacterial strains, Lett. Drug Des. Discovery 4 (2007) 297-304.

[6] I.A.J.M. Bakker-Woudenberg, T. Marian, L. Guo, P. Working, J.W. Mouton, Improved efficacy of ciprofloxacin administered in polyethylene glycol-coated liposomes for treatment of Klebsiella pneumoniae pneumonia in rats, Antimicrob. Agents Chemother. 45 (2001) 1487-1492.

[7] Z. Drulis-Kawa, A. Dorotkiewicz-Jach, Liposomes as delivery systems for antibiotics, Int. J. Pharm. 387 (2010) 187-198.

[8] J.T. McConville, N. Patel, N. Ditchburn, M.J. Tobyn, J.N. Staniforth, P. Woodcock Use of a novel modified TSI for the evaluation of controlled-release aerosol formulations. I, Drug Dev. Ind. Pharm. 26 (2000) 1191-1198. 
[9] R.O. Salama, D. Traini, H.K. Chan, P.M. Young, Preparation and characterisation of controlled release co-spray dried drug-polymer microparticles for inhalation 2: evaluation of in vitro release profiling methodologies for controlled release respiratory aerosols, Eur. J. Pharm. Biopharm. 70 (2008) 145-152.

[10] I. Gonda, Drugs administered directly into the respiratory tract: modeling of the duration of effective drug levels, J. Pharm. Sci. 77 (1988) 340-346.

[11] H.X. Ong, D. Traini, P.M. Young, Pharmaceutical applications of the Calu-3 lung epithelia cell line, Exp. Opin. Drug Deliv. (2013) 1-16.

[12] R. Salama, D. Traini, H.K. Chan, P.M. Young, Recent advances in controlled release pulmonary therapy, Curr. Drug Deliv. 6 (2009) 404-414.

[13] P. Saarinen-Savolainen, T. Järvinen, H. Taipale, A. Urtti, Method for evaluating drug release from liposomes in sink conditions, Int. J. Pharm. 159 (1997) 2733.

[14] M. Beck-Broichsitter, J. Gauss, T. Gessler, W. Seeger, T. Kissel, T. Schmehl, Pulmonary targeting with biodegradable salbutamol-loaded nanoparticles, J. Aerosol Med. Pulm. Drug Deliv. 23 (2010) 47-57.

[15] M. Nagarsenker, V. Londhe, Preparation and evaluation of a liposomal formulation of sodium cromoglicate, Int. J. Pharm. 251 (2003) 49-56.

[16] J. Widdicombe, Regulation of the depth and composition of airway surface liquid, J. Anat. 201 (2002) 313-318.

[17] H. Fischer, J.H. Widdicombe, Mechanisms of acid and base secretion by the airway epithelium, J. Membr. Biol. 211 (2006) 139-150.

[18] H.X. Ong, D. Traini, M. Bebawy, P.M. Young, Epithelial profiling of antibiotic controlled release respiratory formulations, Pharm. Res. (2011) 1-12.

[19] M. Haghi, R. Salama, D. Traini, M. Bebawy, P.M. Young, Modification of disodium cromoglycate passage across lung epithelium in vitro via incorporation into polymeric microparticles, AAPS J. (2011) 1-8.

[20] H.X. Ong, D. Traini, D. Cipolla, I. Gonda, M. Bebawy, H. Agus, P.M. Young, Liposomal nanoparticles control the uptake of ciprofloxacin across respiratory epithelia, Pharm. Res. (2012) 1-12.

[21] A. Tronde, C. Bosquillon, B. Forbes, The isolated perfused lung for drug absorption studies, in: C. Ehrhardt, K.J. Kim (Eds.), Drug Absorption Studies In situ, In vitro and In silico Models, Springer, New York, 2008, pp. 135-163.

[22] M. Sakagami, In vivo, in vitro and ex vivo models to assess pulmonary absorption and disposition of inhaled therapeutics for systemic delivery, Adv. Drug Deliv. Rev. 58 (2006) 1030-1060.

[23] D. Yim, J.D. Blanchard, S. Mudumba, S. Eastman, K. Manda, T. Redelmeier, S.J. Farr, The development of inhaled liposome-encapsulated ciprofloxcin to treat cystic fibrosis, in: R.N. Dalby, P.R. Byron, J. Peart, J.D. Suman, S.J. Farr (Eds.), Respiratory Drug Delivery X, Davis Healthcare Int'l Publishing LCC, River Grove, Boca Raton, Florida, 2006, pp. 425-428.

[24] M.S. Webb, N.L. Boman, D.J. Wiseman, D. Saxon, K. Sutton, K.F. Wong, P. Logan, M.J. Hope, Antibacterial efficacy against an in vivo Salmonella typhimurium infection model and pharmacokinetics of a liposomal ciprofloxacin formulation, Antimicrob. Agents Chemother. 42 (1998) 45.

[25] M. Haghi, P.M. Young, D. Traini, R. Jaiswal, J. Gong, M. Bebawy, Time- and passage-dependent characteristics of a Calu-3 respiratory epithelial cell model, Drug Dev. Ind. Pharm. 36 (2010) 1207-1214.

[26] C. Grainger, L. Greenwell, G. Martin, B. Forbes, The permeability of large molecular weight solutes following particle delivery to air-interfaced cells that model the respiratory mucosa, Eur. J. Pharm. Biopharm. 71 (2009) 318-324.
[27] C.I. Grainger, L.L. Greenwell, D.J. Lockley, G.P. Martin, B. Forbes, C.I. Grainger, L.L. Greenwell, D.J. Lockley, G.P. Martin, B. Forbes, Culture of Calu-3 cells at the air interface provides a representative model of the airway epithelial barrier, Pharm. Res. 23 (2006) 1482-1490.

[28] M. Madlova, C. Bosquillon, D. Asker, P. Dolezal, B. Forbes, In-vitro respiratory drug absorption models possess nominal functional P-glycoprotein activity, J. Pharm. Pharmacol. 61 (2009) 293-301.

[29] K. Hutchison, A.L. Po, I. Gonda, Numerical analysis of approximations to the planar diffusion equation, J. Pharm. Pharmacol. 33 (1981) 46P.

[30] J. Siepmann, N.A. Peppas, Higuchi equation: derivation, applications, use and misuse, Int. J. Pharm. 418 (2011) 6-12.

[31] D.C. Cipolla, F. Dayton, S. Fulzale, E. Gabatan, S. Mudumba, D. Yim, H. Wu, R. Zwilinski, Inhaled liposomal ciprofloxacin: in vitro properties and aerosol performance, in: R.N. Dalby, P.R. Byron, J. Peart, J.D. Suman, S.J. Farr, P.M. Young (Eds.), Respiratory Drug Delivery, Davis Healthcare Int'l Publishing, River Grove, Orlando Florida, 2010, pp. 409-414.

[32] N.R. Mathias, J. Timoszyk, P.I. Stetsko, J.R. Megill, R.L. Smith, D.A. Wall, Permeability characteristics of calu-3 human bronchial epithelial cells: in vitro-in vivo correlation to predict lung absorption in rats, J. Drug Target. 10 (2002) 31-40.

[33] A. Tronde, B. Nordén, A.B. Jeppsson, P. Brunmark, E. Nilsson, H. Lennernäs, U.H. Bengtsson, Drug absorption from the isolated perfused rat lung-correlations with drug physicochemical properties and epithelial permeability, J. Drug Target. 11 (2003) 61-74.

[34] F. Manford, A. Tronde, A.B. Jeppsson, N. Patel, F. Johansson, B. Forbes, Drug permeability in 16HBE140-airway cell layers correlates with absorption from the isolated perfused rat lung, Eur. J. Pharm. Sci. 26 (2005) 414-420.

[35] A. Tronde, B. Nordén, H. Marchner, A.K. Wendel, H. Lennernäs, U.H. Bengtsson, Pulmonary absorption rate and bioavailability of drugs in vivo in rats: structure, Äìabsorption relationships and physicochemical profiling of inhaled drugs, J. Pharm. Sci. 92 (2003) 1216-1233.

[36] M.E. Cavet, M. West, N.L. Simmons, Transepithelial transport of the fluoroquinolone ciprofloxacin by human airway epithelial Calu-3 cells, Antimicrob. Agents Chemother. 41 (1997) 2693-2698.

[37] C. Bosquillon, Drug transporters in the lung, do they play a role in the biopharmaceutics of inhaled drugs?, J Pharm. Sci. 99 (2010) 2240-2255.

[38] H.X. Ong, D. Traini, M. Bebawy, P.M. Young, Ciprofloxacin is actively transported across bronchial lung epithelial cells using a Calu-3 air interface cell model, Antimicrob. Agents Chemother. 57 (2013) 2535-2540.

[39] B. Forbes, C. Ehrhardt, Human respiratory epithelial cell culture for drug delivery applications, Eur. J. Pharm. Biopharm. 60 (2005) 193-205.

[40] F. Komada, S. Iwakawa, N. Yamamoto, H. Sakakibara, K. Okumura, Intratracheal delivery of peptide and protein agents: absorption from solution and dry powder by rat lung, J. Pharm. Sci. 83 (1994) 863-867.

[41] B.L. Laube, G.W. Benedict, A.S. Dobs, Time to peak insulin level, relative bioavailability, and effect of site of deposition of nebulized insulin in patients with noninsulin-dependent diabetes mellitus, J. Aerosol Med. 11 (1998) 153173.

[42] S.-A. Cryan, N. Sivadas, L. Garcia-Contreras, In vivo animal models for drug delivery across the lung mucosal barrier, Adv. Drug Deliv. Rev. 59 (2007) 1133-1151. 